\title{
Chromium-modified goethite in an external magnetic field
}

\author{
E van den Pol ${ }^{1}$, D M E Thies-Weesie ${ }^{1}$, A V Petukhov ${ }^{1}$, P Panine Pan $^{2,3}$ \\ and G J Vroege ${ }^{1}$ \\ ${ }^{1}$ Van 't Hoff Laboratory for Physical and Colloid Chemistry, Debye Institute for \\ Nanomaterials Science, Utrecht University, Padualaan 8, 3584 CH Utrecht, The Netherlands \\ ${ }^{2}$ High Brilliance Beamline ID2, European Synchrotron Radiation Facility, BP 220, \\ 38043 Grenoble, France \\ E-mail: e.vandenpol@uu.nl
}

Received 28 March 2008, in final form 28 May 2008

Published 10 September 2008

Online at stacks.iop.org/JPhysCM/20/404219

\begin{abstract}
The behaviour of the different liquid crystalline phases observed in colloidal dispersions of goethite has been studied by small angle $\mathrm{x}$-ray scattering. The particles align parallel to a small magnetic field and perpendicular to a large magnetic field, which was observed in the isotropic and nematic phase. The smectic phase also aligned parallel to a small magnetic field but it formed an aligned columnar phase with a distorted hexagonal structure in higher magnetic fields. The columnar phase mainly aligned in higher magnetic fields. Goethite particles were successfully modified with chromium. The Cr-modified goethite particles show similar phase behaviour as normal goethite particles but with different magnetic properties. The relative importance of the permanent and induced magnetic moment was found to have changed.
\end{abstract}

(Some figures in this article are in colour only in the electronic version)

\section{Introduction}

Although organic liquid crystals are ever more widely spread and are well known for their applications such as LCD screens, mineral liquid crystals have an almost equally long history and show enhanced electrical, optical and magnetic properties, together with a better thermal stability [1].

An interesting example are colloidal dispersions of the mineral goethite $(\alpha-\mathrm{FeOOH})$. Special magneto-optical effects of aqueous colloidal suspensions of mixed iron oxides were already recognized by Majorana in 1902 [2] and further studied by Cotton and Mouton in 1907 [3]. It turned out that one of the components was goethite. Goethite can easily form liquid crystals that are susceptible to external fields. Recent investigations by Lemaire et al [4-8] further revealed that the board-like goethite particles have a permanent magnetic moment along their long axis, presumably due to uncompensated spins within their anti-ferromagnetic crystal structure, combined with an induced magnetic moment oriented predominantly along the shortest particle dimension.

\footnotetext{
3 Present address: Xenocs SA, 19 rue Francois Blumet, 38360 Sassenage, France.
}

Therefore, they have opposing tendencies to align with their permanent moment parallel to the field at low magnetic field strength and to orient perpendicular to a higher magnetic field due to their induced moments.

By tuning the relative importance of the permanent and induced moments different phase diagrams can be expected [9]. When the permanent magnetic moment is relatively unimportant compared to the susceptibility anisotropy, the first-order isotropic-nematic phase transition changes into a second-order transition in a large magnetic field. In the other situation, where the permanent moment is more important, the first-order phase transition vanishes above a critical point at a lower field strength and subsequently reappears at higher fields. We hope to achieve these different types of behaviour by modification of the goethite particles with other elements. Goethite has many isostructural equivalents where other cations occupy the places of $\mathrm{Fe}^{3+}[10,11]$. Therefore, it is possible to modify goethite with different elements, for example with chromium $(\mathrm{Cr})[12,13]$, which has been studied here.

Apart from the nematic phase goethite can also form higher order phases. Smectic and columnar phases have been found even in very $(55 \%)$ polydisperse systems $[14,15]$. 
Table 1. Goethite particle dimensions.

\begin{tabular}{llllllll}
\hline \multirow{2}{*}{ System } & $\% \mathrm{Cr}$ & $\begin{array}{l}\langle L\rangle \\
(\mathrm{nm})\end{array}$ & $\begin{array}{l}\sigma_{\mathrm{L}} \\
(\%)\end{array}$ & $\begin{array}{l}\langle W\rangle \\
(\mathrm{nm})\end{array}$ & $\begin{array}{l}\sigma_{W} \\
(\%)\end{array}$ & $\begin{array}{l}\langle T\rangle \\
(\mathrm{nm})\end{array}$ & $\frac{\langle L\rangle}{\langle W\rangle}$ \\
\hline gn & 0 & 282 & 35 & 68 & 32 & $\sim 25$ & 4.1 \\
gcr & 4.6 & 227 & 31 & 68 & 29 & $\sim 22$ & 3.3 \\
\hline
\end{tabular}

Sedimentation and fractionation play an important role in the formation of these phases. All different phases of unmodified and $\mathrm{Cr}$-modified goethite were studied by small angle $\mathrm{x}$-ray scattering (SAXS) in a magnetic field.

\section{Experimental details}

\subsection{Synthesis}

Goethite particles were obtained by hydrolysis of iron nitrate at high $\mathrm{pH}$ according to Lemaire et al [5]. $1 \mathrm{M} \mathrm{NaOH}$ (Merck, p.a.) was added dropwise, under stirring, to a $0.1 \mathrm{M}$ iron nitrate (Acros, p.a.) solution until a $\mathrm{pH}$ of 11-12 was reached. The precipitate was aged for 9 days after which the supernatant was removed and the sediment was washed two times with doubly distilled (dd) water and charged with $3 \mathrm{M} \mathrm{HNO}_{3}$ (Merck, 65\%, p.a.). After centrifuging and redispersing in dd water for three times the particles were redispersed in dd water of $\mathrm{pH}=3$.

The chromium-modified particles were synthesized in a slightly adjusted way with part of the iron nitrate replaced by chromium nitrate (Merck, p.a.). In this case $1 \mathrm{M} \mathrm{NaOH}$ was added to the $0.1 \mathrm{M}$ chromium/iron nitrate solution until a $\mathrm{pH}$ of 9 was reached. Then the precipitate was redispersed in fresh millipore water before raising the $\mathrm{pH}$ further to 11-12. Ageing took place at $70^{\circ} \mathrm{C}$ for 10 days. The obtained dispersion was centrifuged and stabilized as described above.

It is assumed that after many centrifugation steps the salt concentration is determined by the $\mathrm{H}^{+}$and $\mathrm{OH}^{-}$ concentrations. The Debye length is then $10 \mathrm{~nm}$ at most.

\subsection{Characterization}

Particle size distributions were determined by transmission electron microscopy (TEM) using a Technai 10 and 12 (FEI company) electron microscope. The length and width of about 500 particles was measured with iTEM imaging software to determine the average length $(\langle L\rangle)$ and width $(\langle W\rangle)$ and their standard deviation $\left(\delta_{\mathrm{L}}\right.$ and $\left.\delta_{W}\right)$. The length polydispersity is then defined as $\sigma_{\mathrm{L}}=\delta_{\mathrm{L}} /\langle L\rangle$. The thickness was difficult to measure because hardly any particles lay on their sides on the TEM grid. For each sample about 10-20 particle thicknesses were measured.

Scanning transmission electron microscopy (STEM) in combination with energy dispersive $\mathrm{x}$-ray analysis (EDX) was used to do elemental analysis. A 'bulk' analysis was done by averaging measurements over a group of about 20 particles. A line scan over the width of a particle was done to get information about the distribution of $\mathrm{Cr}$ over the particles.

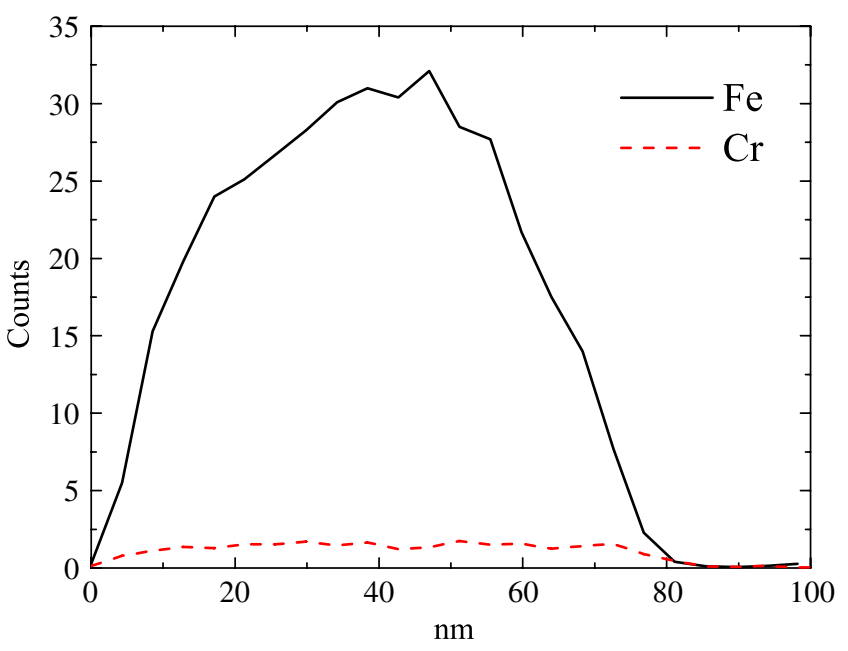

Figure 1. EDX line profile along the width of a Cr-modified goethite particle.

\subsection{SAXS experiments}

Samples with different volume fractions were prepared in capillaries (Vitrocom RT3524) with internal dimensions of $0.2 \times 4.0 \times 10 \mathrm{~mm}^{3}$. The capillaries were closed with two-component epoxy glue (Bison Kombi rapide) and kept in a vertical position to allow the establishment of the sedimentation equilibrium profile. The normal goethite sample had been standing for about 3.5 years before doing these measurements. The $\mathrm{Cr}$-modified goethite sample was about a year old and had been measured in a magnetic field two months before doing these measurements. SAXS measurements were performed at the ID2 beamline of the European Synchrotron Radiation Facility (ESRF, Grenoble, France), which is characterized by a narrow collimated beam to scan the capillary, low q value, high sensitivity and high detector dynamic. A variable permanent magnet was used, with which a magnetic field up to $1.5 \mathrm{~T}$ could be reached [5]. Two stacks of NdFeB permanent magnets generated the field and the distance between them could be adjusted to reach the desired field strength. Different stacks were available, one set gave a field perpendicular to the $\mathrm{x}$-ray beam and another set a field parallel to the x-ray beam. So, by changing the stacks also the direction of the magnetic field could be altered.

\section{Results and discussion}

\subsection{Characterization}

The particle dimensions of the systems used are shown in table 1 .

With EDX a 'bulk' molar concentration of chromium $([\mathrm{Cr}] /([\mathrm{Fe}]+[\mathrm{Cr}]))$ of $4.6 \%$ was found. The line profile along the width of the particle, which is shown in figure 1 , shows that the $\mathrm{Cr}$ is homogeneously distributed over the goethite particle.

\subsection{Phase behaviour of normal goethite in a magnetic field}

The goethite system studied here shows rich phase behaviour. It forms an isotropic (I), nematic (N), smectic (S) and columnar 

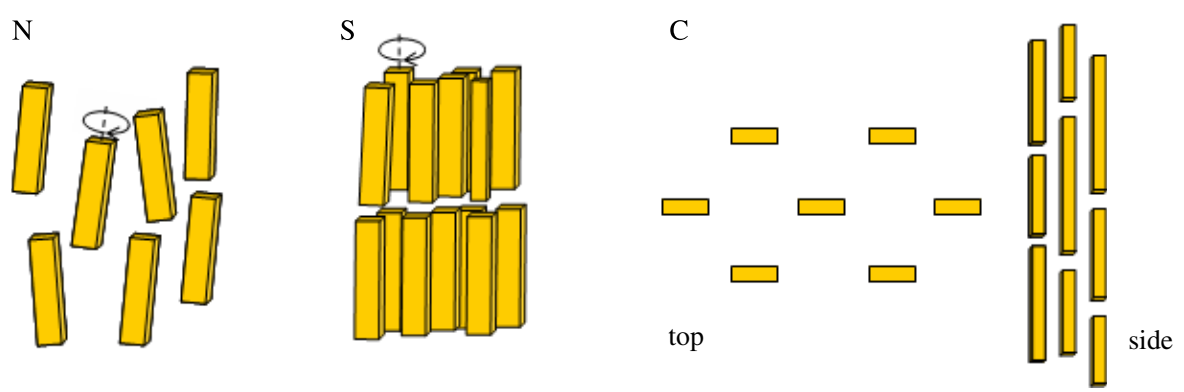

Figure 2. Structure of the nematic $(\mathrm{N})$, smectic $(\mathrm{S})$ and columnar $(\mathrm{C})$ phase of goethite. For the columnar phase a top and a side view are shown. The particles are drawn in one orientation but in the nematic and smectic phase they can rotate around their long axis.

(C) phase in a sample with an initial volume fraction of $5.8 \%$. The structure of the different phases can be seen in figure 2 . This sample had been standing for a long time and therefore sedimentation and fractionation contributed to the formation of the higher order phases [14]. A polarization microscopy picture is shown in figure 3 . The lowest phase is a combined smectic and columnar phase $(C+S)$ of which the upper part is purely smectic. On top of this phase a nematic phase can be seen and the upper phase is isotropic. The dark part at the bottom of the picture is the glue which was used to close the capillary. An estimate of the volume fraction at the phase boundaries has been made from the X-ray intensities measured during the SAXS experiments. The volume fraction at the I$\mathrm{N}$ phase boundary is approximately $7.3 \%$, at N-S $10 \%$ and at $\mathrm{S}-(\mathrm{C}+\mathrm{S}) 11 \%$. No clear jumps in the concentration profile were observed at the phase boundaries. All different phases were studied with SAXS in an external magnetic field.

3.2.1. Nematic phase. Lemaire et al [4] already found that goethite particles align parallel to a small magnetic field and perpendicular to a large magnetic field. This is also shown in figure 4 for the nematic phase. In the upper part of the figures (a)-(c) the magnetic field is perpendicular to the X-ray beam (in the vertical direction) and in the lower part of the figures (d)-(f) the magnetic field is parallel to the x-ray beam. All measurements with the field perpendicular to the beam were performed before the measurements with the field parallel to the beam. The difference between the patterns shown in (a) and (d), which were both measured without external magnetic field, illustrate the effect of the sample history. The application of the magnetic field perpendicular to the beam (a)-(c) and the quick removal of the field leads to a different distribution of orientations of the nematic domains. Also, the influence of a weak residual magnetic field of a few $\mathrm{mT}$ cannot be completely excluded.

It can be seen that without a magnetic field the nematic phase is already mostly oriented in one domain (figure 4(a), the orientation is similar to figure $2(\mathrm{~N})$ ). The diffuse peaks at large angle originate from the liquid-like order perpendicular to the direction of orientation in the nematic phase. At small angles there are also peaks visible (partly screened by the beamstop) indicating positional correlations on distances of the order of the length of the particles. However these peaks are quite broad and cannot originate from a smectic phase. So in

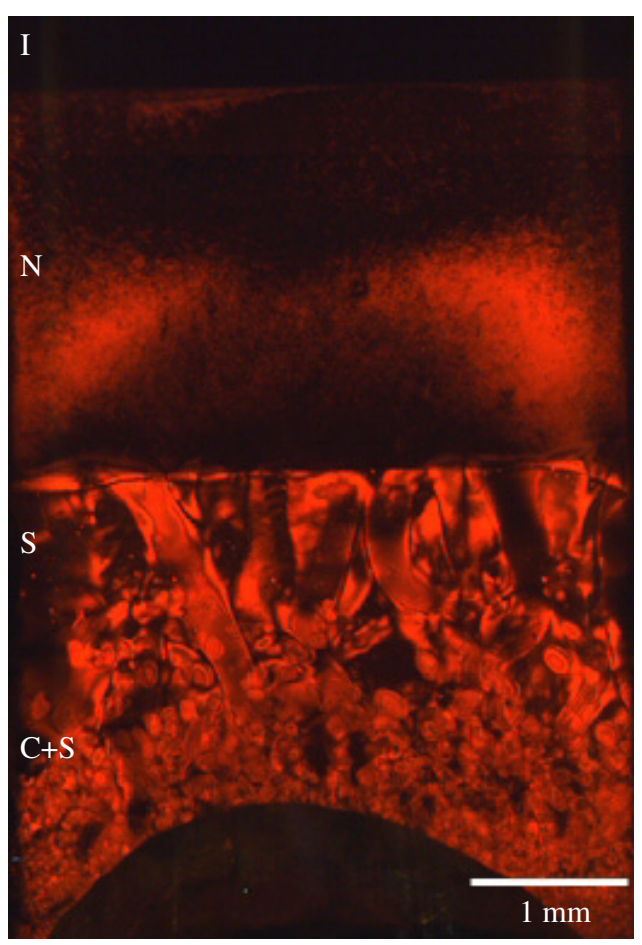

Figure 3. Liquid crystalline phases observed between crossed polarizers in a goethite sample.

this system there are already strong length correlations in the nematic phase.

In a magnetic field of $0.1 \mathrm{~T}$ the particles in the nematic phase are clearly better aligned parallel to the field (b). The SAXS pattern with the field parallel to the beam (e) shows an isotropic ring meaning that the shortest two axes of the particles do not have a preferred orientation.

At $1.5 \mathrm{~T}$ the particles change orientation and are now aligned perpendicular to the field (c). In the patterns (c) and (f) weak scattering can be observed at small angles, perpendicular to the field. This means that length correlations are present perpendicular to the magnetic field. In the picture with the field parallel to the beam (f) it can be seen that the isotropic ring is now at a significantly smaller angle than in a small magnetic field. The $q$-values correspond to $136 \mathrm{~nm}$ compared to $91 \mathrm{~nm}$ in a small magnetic field. This means that within a plane perpendicular to the field the particles are further apart from 


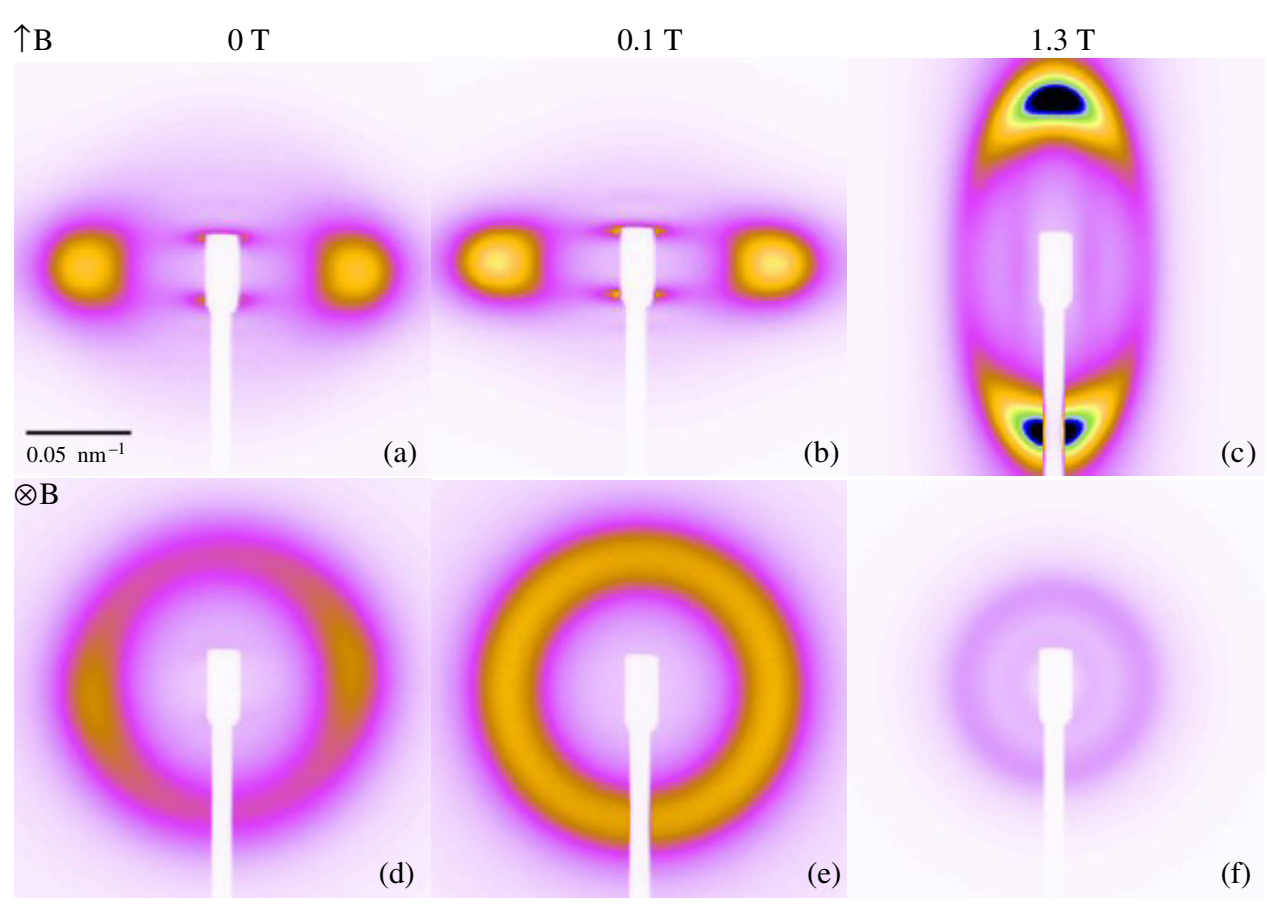

Figure 4. Nematic phase of goethite in a magnetic field. Upper pictures with the magnetic field perpendicular to the beam (in the vertical direction), lower pictures with the field parallel to the beam.

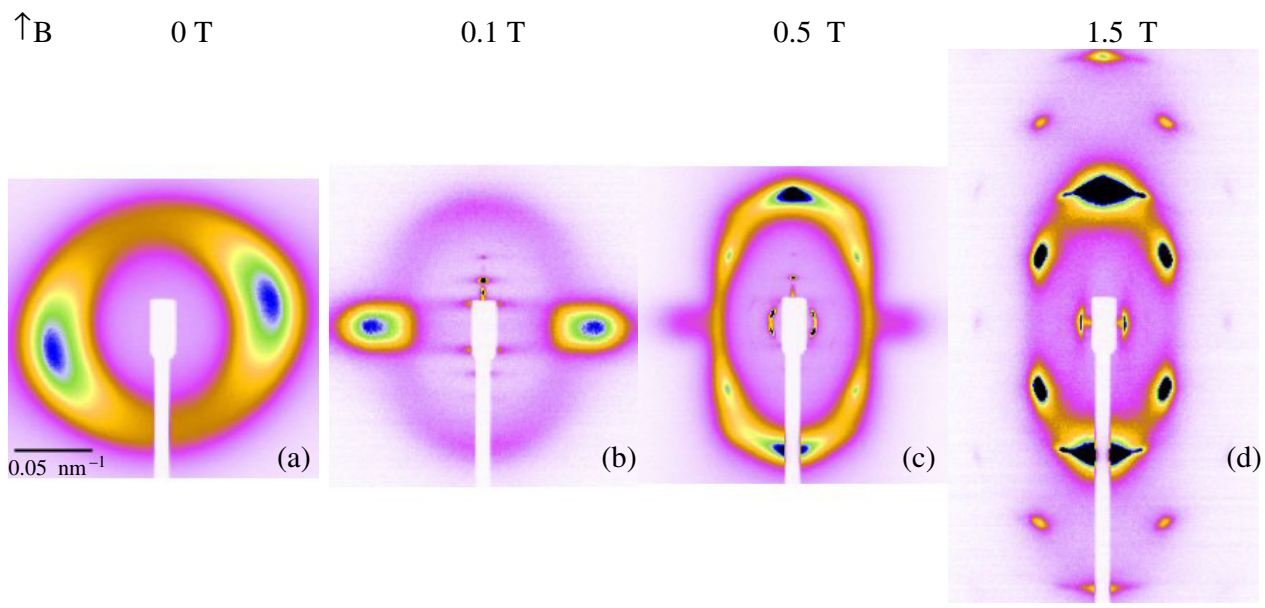

Figure 5. Smectic phase of goethite in a magnetic field.

each other. From the picture with the field perpendicular to the beam (c) a distance of $73 \mathrm{~nm}$ along the field can be calculated which is smaller than the $91 \mathrm{~nm}$ in small fields, so now shorter distances are present along the field. This means that the particles are mainly oriented with their shortest dimension along the field and their two larger dimensions perpendicular to the field.

3.2.2. Smectic phase. The behaviour of the smectic phase in a magnetic field is shown in figure 5. Without a magnetic field the typical sharp peaks at small angles, corresponding to the smectic periodicity of the layers, are not observed (a). At the same time, optical Bragg reflections were observed in the sample at this height. The apparent discrepancy can be understood on the basis of the orientations of the smectic domains present in the sample. To observe the smectic periodicity in a SAXS pattern the X-ray beam must be parallel to the smectic layers. A slight tilt of the layers is already enough to make the small angle peaks disappear.

In a small magnetic field of $0.1 \mathrm{~T}$ the smectic phase aligns with the particles along the magnetic field (b). The small angle peaks corresponding to the periodicity of the layers can now be seen, even up to the third order. The broad peaks originate from the liquid-like interactions within the smectic layers. They are now less spread out than without a field, indicating an alignment with the field. At a field strength of $0.5 \mathrm{~T}$ the smectic phase starts to transform into a columnar phase with a distorted hexagonal structure (c, structure-like figure 2(C)), 


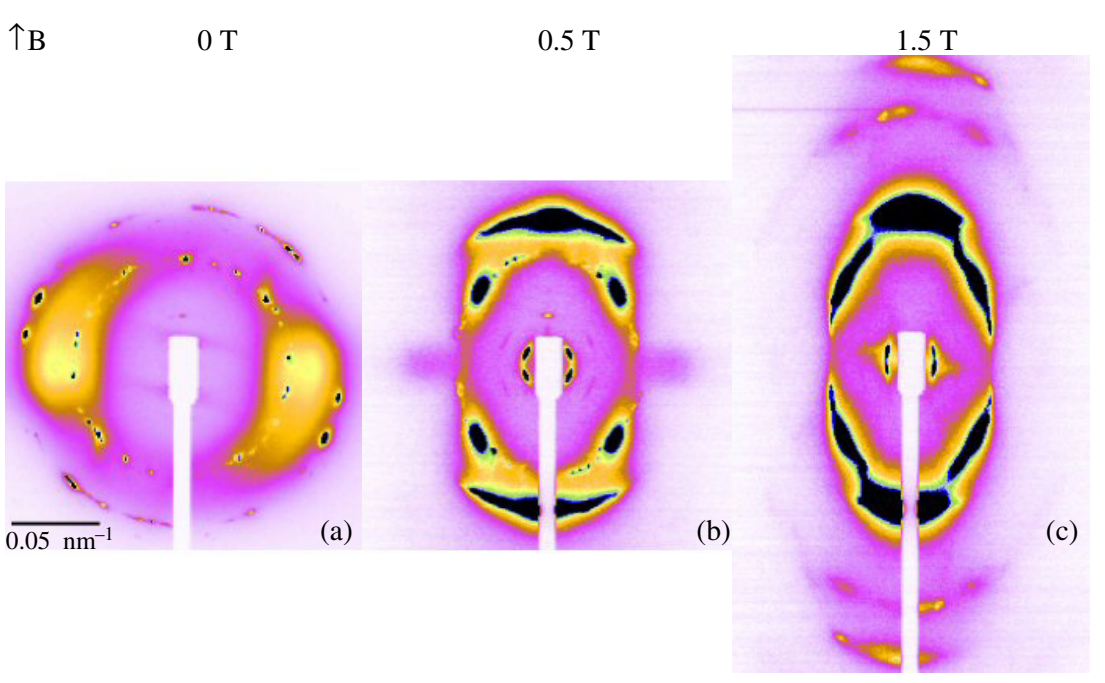

Figure 6. Combined columnar and smectic phase of goethite in a magnetic field.

as was earlier observed for a nematic phase in a magnetic field [7]. At small angles there are smectic peaks that are oriented in multiple directions indicating that this is not an equilibrium structure yet. At the highest field strength of $1.5 \mathrm{~T}$ the columnar structure is nicely ordered (d), second-order peaks can be observed (even third-order peaks were observed with a very small intensity). Higher order peaks are mainly observed in the direction of the field. This is because the structure is formed in all perpendicular directions and the diffraction spots are actually cross sections through scattering rings around the field direction. The peaks at small angles suggest that there are still remnants of the smectic phase present, now oriented with its layers perpendicular to the field.

\subsubsection{Columnar phase. No pure columnar phase was found} in this system, so the columnar phase was studied together with the smectic phase. The behaviour in a magnetic field of those two phases together can be seen in figure 6 . The powder-like scattering rings are typical for a columnar phase with a lot of differently oriented domains. The much broader liquid-like peak of the smectic phase lies in between the columnar rings. Hardly any change of the diffraction pattern was observed in a small magnetic field. At $0.5 \mathrm{~T}$ (b) it can be seen that the long axis of the particles is mainly oriented perpendicular to the field, as in figure 2(C). The domains tend to orient in the field but still some of the powder-like peaks can be observed and at small angles the smectic peaks are in some intermediate state. At $1.5 \mathrm{~T}$ (c) the structure is more ordered but it is clear that it is not as nice as the columnar structure developed from the smectic phase discussed before (see figure 5(d)). It seems that this part of the sample which is almost at the bottom of the capillary cannot rearrange that easily. It is probably more restricted because of the higher osmotic pressure.

\subsection{Influence of incorporated chromium}

To tune the magnetic properties of goethite a part of its $\mathrm{Fe}$ was replaced by $\mathrm{Cr}$. This sample contains $4.6 \%$ (mol) $\mathrm{Cr}$. In a capillary with an initial volume fraction of $7.8 \%$ the same phases were found as for the pure goethite sample. The estimated volume fraction at the I-N phase boundary is approximately $12 \%$, at $\mathrm{N}-\mathrm{S} 13.4 \%$ and at $\mathrm{S}-(\mathrm{C}+\mathrm{S}) 14.4 \%$. No clear jumps in the concentration profile were observed at the phase boundaries.

In a small magnetic field of $0.1 \mathrm{~T}$ the system shows the same behaviour as pure goethite; the isotropic, nematic and smectic phase are aligned parallel to the field. The combined columnar and smectic phases further down in the sample have not yet reacted to the magnetic field.

On increasing the magnetic field to $0.5 \mathrm{~T}$ differences between the normal and Cr-modified goethite become visible (figure 7). In the upper part of the picture normal goethite (gn, (a)-(d)) is shown and in the lower part Cr-modified goethite (gcr, (e)-(h)). The isotropic and nematic phases of gn are already mostly oriented perpendicular to the field ((a), (b)) as opposed to gcr which is still predominantly oriented parallel to the field ((e), (f)), although a very small part of the system did turn perpendicular. When the smectic phase of gn starts to form an ordered columnar phase (c), the smectic phase of gcr is still mostly aligned with the field (g). The combined columnar and smectic phases of gn (d) start to form an ordered columnar phase just like the pure smectic phase (c). But the columnar phase within the combined smectic and columnar phase of gcr has hardly changed and its smectic part is still mostly aligned parallel to the field (h). The columnar phase of gcr is slightly unusual because three rings are observed instead of the two which are observed for normal goethite. This suggests that another kind of columnar structure is formed, probably a rectangular structure where a distorted hexagonal structure (as in figure $2(\mathrm{C})$ ) is normally found. This is currently a subject of further study.

At $1.5 \mathrm{~T}$ it can be seen that the gcr system eventually does show the same behaviour as normal goethite (figure 8). The isotropic and nematic phase are now aligned perpendicular to the field ((a), (b) as in figure 4(c)). In the smectic phase there are actually two different effects observed. High in the smectic phase (c) the system stays smectic but it aligns perpendicular to the field, whereas low in the smectic phase (d) it forms an 

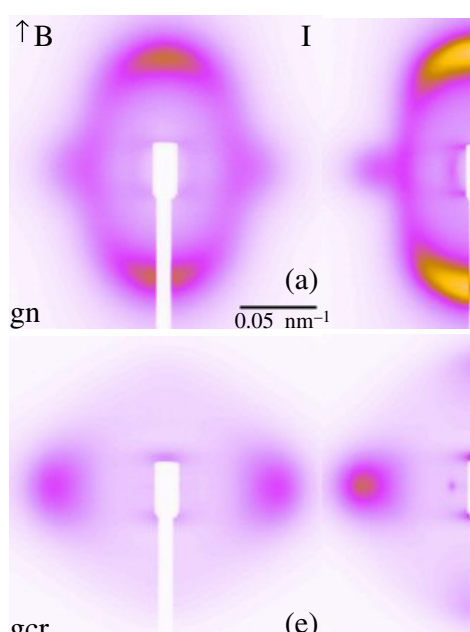

$\mathrm{gcr}$
$\mathrm{N}$

(b)

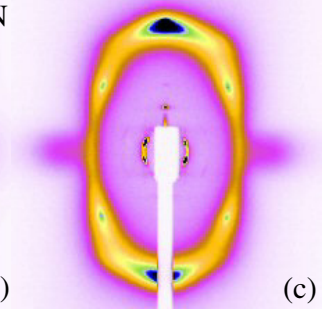

c)
$\mathrm{S}+\mathrm{S}$

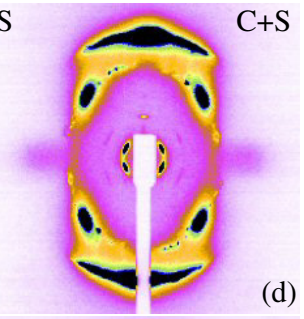

(d)

Figure 7. Cr-modified goethite compared to normal goethite in a magnetic field of $0.5 \mathrm{~T}$.
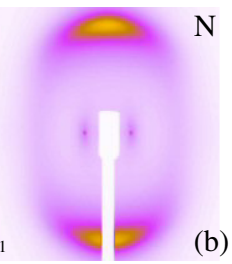

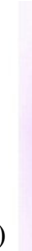

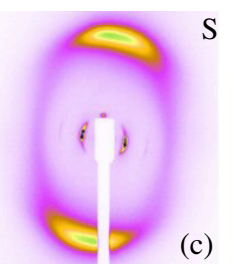
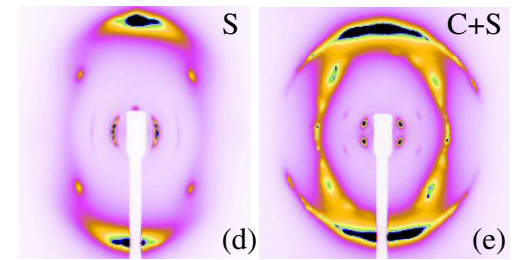

Figure 8. Cr-modified goethite in a magnetic field of $1.5 \mathrm{~T}$.

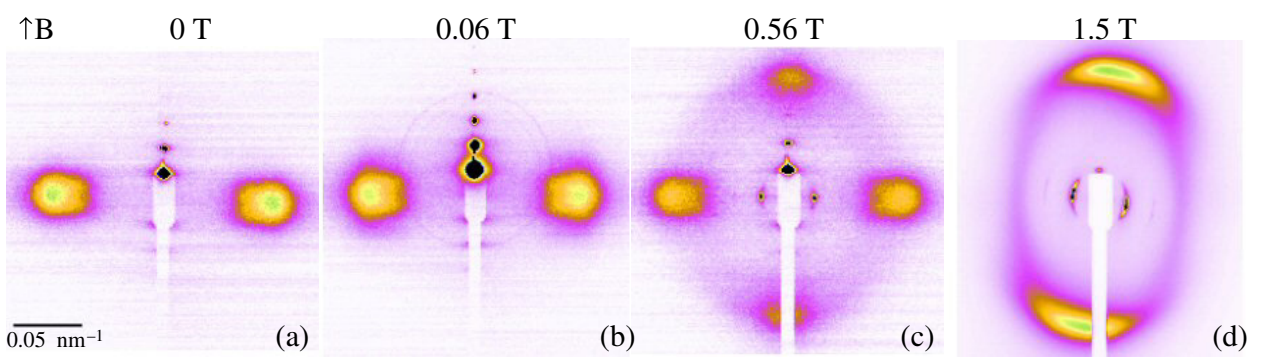

Figure 9. Smectic phase of Cr-modified goethite in a magnetic field.

ordered columnar phase as was observed for gn (figures 5(c), (d)). It seems that either the columnar phase which is already present close to the lower part of the smectic phase induces the formation of this ordered columnar phase, or the higher osmotic pressure at this height favours the transformation to a columnar phase. The columnar phase itself also starts to orient (e) but it probably needs more time to align further.

3.3.1. Highly ordered smectic phase. The smectic phase in the Cr-modified system shows remarkable behaviour (figure 9). It is already pretty well aligned without a magnetic field (a): a third-order peak can be observed. This is possibly because of preliminary measurements carried out two months before doing these measurements where the sample had been aligned in a magnetic field. In a magnetic field of only $60 \mathrm{mT}$ (b) it even shows a sixth-order peak which indicates a very well ordered smectic phase. Very short measurement times of a few milliseconds were needed together with a filter so as not to oversaturate the detector. A longer measurement time was used to visualize the higher order peaks, thereby slightly oversaturating the lower order peaks and causing some artefacts in the SAXS pattern (figure 9(b)).

On increasing the field the order slowly decreases and at $0.56 \mathrm{~T}$ the particles start to turn perpendicular to the field (c). At $1.5 \mathrm{~T}$ the smectic phase is mostly oriented perpendicular to the field (d); a second-order small angle peak can be observed. But there are still some different domains as can be seen from the peaks at small angle. A higher field or longer exposure to the field are probably needed to fully align the phase.

\section{Conclusions}

Goethite liquid crystalline phases show interesting behaviour in a magnetic field. The nematic phase aligns parallel to the 
field at low field strength and perpendicular to a large magnetic field, with the shortest axis mainly oriented along the field. The smectic phase also aligns parallel to a small magnetic field but it forms a columnar phase with a distorted hexagonal structure in a higher magnetic field. The behaviour of the columnar phase could only be studied together with a smectic phase. This combination has also the tendency to form an oriented columnar phase in a magnetic field but it reacts more slowly probably because the particles seem to be more restricted at higher osmotic pressure.

Goethite particles were successfully modified with $\mathrm{Cr}^{3+}$ ions, which are incorporated homogeneously. The modified particles show the same phase behaviour as normal goethite. Furthermore, in a magnetic field similar effects are observed. The difference is that the particles turn perpendicular to the field at a considerably higher magnetic field strength. This proves that the relative importance of the permanent and induced magnetic moment can indeed be changed by incorporating different metal ions into the goethite particles. The effect of modification by other elements like Al and Co will be a subject of further study.

\section{Acknowledgments}

This work is part of the research programme SFB TR6 of the 'Stichting voor Fundamenteel Onderzoek der Materie (FOM)', which is financially supported by the 'Nederlandse Organisatie voor Wetenschappelijk Onderzoek (NWO)' and 'Deutsche Forschungsgemeinschaft (DFG)'. The work of DME T-W was performed as part of a NWO-CW TOP project. The staff of the ID2 beamline at ESRF is acknowledged for giving us the opportunity to measure at their beamline and giving their support, in particular $\mathrm{T}$ Narayanan for fruitful discussions. EDX was carried out in collaboration with EMU, the Electron Microscopy group at Utrecht University, the Netherlands.

\section{References}

[1] Gabriel J C P and Davidson P 2000 Adv. Mater. 12 9-20

[2] Majorana Q 1902 Rend. Accad. Linc. 11-1 374

[3] Cotton A and Mouton H 1907 Ann. Chim. Phys. 11145

[4] Lemaire B J, Davidson P, Ferré J, Jamet J P, Panine P, Dozov I and Jolivet J P 2002 Phys. Rev. Lett. 88125507

[5] Lemaire B J, Davidson P, Ferré J, Jamet J P, Petermann D, Panine P, Dozov I and Jolivet J P 2004 Eur. Phys. J. E 13 291-308

[6] Lemaire B J, Davidson P, Petermann D, Panine P, Dozov I, Stoenescu D and Jolivet J P 2004 Eur. Phys. J. E 13 309-19

[7] Lemaire B J, Davidson P, Panine P and Jolivet J P 2004 Phys. Rev. Lett. 93267801

[8] Lemaire B J, Davidson P, Ferré J, Jamet J P, Petermann D, Panine P, Dozov I, Stoenescu D and Jolivet J P 2005 Faraday Discuss. 128 271-83

[9] Wensink H H and Vroege G J 2005 Phys. Rev. E 72 031708-9

[10] Cornell R M and Schwertmann U 1996 The Iron Oxides (Weinheim: VCH)

[11] Schwertmann U and Cornell R M 2000 Iron Oxides in the Laboratory: Preparation and Characterization 2nd edn (Weinheim: Wiley-VCH)

[12] Schwertmann U, Gasser U and Sticher H 1989 Geochim. Cosmochim. Acta 53 1293-7

[13] Sileo E E, Ramos A Y, Magaz G E and Blesa M A 2004 Geochim. Cosmochim. Acta 68 3053-63

[14] Vroege G J, Thies-Weesie D M E, Petukhov A V, Lemaire B J and Davidson P 2006 Adv. Mater. 18 2565-8

[15] Thies-Weesie D M E, de Hoog J P, Hernandez Mendiola M H, Petukhov A V and Vroege G J 2007 Chem. Mater. $195538-46$ 